\title{
Relapsed neuroblastoma in the central nervous system, a single-center, retrospective study
}

\author{
Yutong Zhang ${ }^{1}$, Xianmei Jin ${ }^{1}$, Chao Zhang ${ }^{1}$, Dan Zhong ${ }^{1}$, and Jian Chang ${ }^{1}$ \\ ${ }^{1}$ Jilin University First Hospital
}

November 13, 2020

\begin{abstract}
Background: The incidence of relapsed neuroblastoma (NB) in the central nervous system (CNS) is less than $20 \%$. Here, we presented eleven cases with CNS relapsed NB to better understand the CNS relapsed NB. Methods: Medical records from NB patients between January 1st, 2010 and December 31st, 2017 were reviewed to examine those diagnosed with CNS relapsed NB. The following patient information was extracted, including age, gender, histological subtyping, tumor stage, risk group, biomarkers, chemotherapy regimen and dosage, surgery details, treatment outcomes and follow-up, clinical manifestations of CNS relapsed NB, treatment protocols for CNS relapsed NB, and patient outcomes (including the cause of death). Results: From January 1st, 2010 to December 31st, 2017, eleven NB patients were diagnosed with CNS relapse at our department. All the eleven patients were classified to have high risk disease. The median time from the completion of chemotherapy to CNS relapse was 5 months. Five patients did not receive any treatment for the relapsed disease and finally died of it. Curative treatments were tried in six patients. At last, two patients with isolated CNS relapsed NB survived. Conclusions: The median time from the completion of chemotherapy to CNS relapse is very short in the present retrospective study. It seems that patients with isolated CNS relapsed disease have better outcomes. We suggest the temozolomide-topotecan combination as an add-one backbone for further salvage treatment in children with CNS relapsed NB, even though this combination is not superior to others in terms of its efficacy.
\end{abstract}

\section{Introduction}

Neuroblastoma (NB) is the most common extracranial solid tumor in children, and over $1 / 2 \mathrm{NB}$ children develop distant metastasis at the time of diagnosis. The most common sites of metastasis include bone, bone marrow, liver, and skin[1]. With regard to the relapsed pattern, local foci (8\%), primary metastatic sites $(66 \%)$, and primary site in addition to metastatic sites (26\%) are the major patterns[2]. Metastasis in the central nervous system (CNS) is rare on presentation, let alone the CNS relapsed NB. Little is known on the clinical characteristics of CNS relapsed NB, and its incidence is less than 20\% [3, 4]. Over the past 10 years, our department have admitted several CNS relapsed NB cases without CNS involvement at the initial diagnosis. Relapsed CNS NB is associated with poor outcomes, with a 2-year event-free survival (EFS) of $10 \%$ and a 4-year overall survival (OS) of 20\%[5]. Herein, we reported a small case series of CNS relapsed NB to better understand CNS relapsed NB.

\section{Methods}

Our study protocol was approved by the Ethical Institution of our hospital. Medical records from NB patients between January $1^{\text {st }}, 2010$ and December $31^{\text {st }}, 2017$ were reviewed to identify patients diagnosed with CNS relapsed NB.

The diagnosis was made and the stage was assessed according to criteria of the International Neuroblastoma Staging System (International Neuroblastoma Staging System, INSS)[6-8]. Typically, a tissue diagnosis of NB can be made based on conventional histology (with or without immunohistology and increased urine or 
serum catecholamines or metabolites). In addition, a diagnosis can also be made when unequivocal tumor cells are detected in bone marrow samples and increased urine or serum catecholamines or metabolites are present [9].

The following data were extracted from each patient, namely, age, gender, histological subtyping, tumor stage, risk group, biomarkers, chemotherapy regimen and dosage, surgery details, treatment outcomes and follow-up, clinical manifestations of CNS relapsed NB, treatment protocols for CNS relapsed NB, and patient outcomes (including the cause of death). Data collection from the clinical records of patients was approved by the Institutional Review Boards. Additionally, written informed consents were obtained from the parents or legal guardians of all patients before the initiation of treatment.

\section{Results}

From January $1^{\text {st }}, 2010$ to December $31^{\text {st }}, 2017$, altogether eleven NB patients were diagnosed with CNS relapse at our department. The median age at diagnosis was 4 (range, 2-8) years. More details about the characteristics of these eleven patients are presented in Table 1. Among these eleven patients, two had stage III disease while nine had stage IV disease at the initial diagnosis, and all of them were classified to have high risk disease. The two patients with high-risk stage III disease showed unfavorable histology, and one patient with stage IV disease was detected with $M Y C N$ amplification. Patients investigated in this study received chemotherapy in accordance with the NB97 trial in combination with a novel delayed intensification chemotherapy $[9,10]$. If feasible, surgery was performed after the completion of cycle 4 or 6 chemotherapy before initiating the N7 treatment. Gross total resection of the primary tumor was assessed by computed tomography $(\mathrm{CT}$ ) or magnetic resonance imaging (MRI). As for CNS relapsed NB, the diagnosis was made by enhanced head CT or MRI.

The time to CNS relapse was short, which was no more than 6 months (range, 1-9 months; median, 5 months) after the completion of delayed intensification chemotherapy in eight of the eleven $(72.7 \%)$ patients. For patients with CNS relapse, five had relapsed disease in the CNS alone (including 4 in parenchyma and 1 in leptomeninges), while seven had systemic bony and marrow disease in CNS relapse. Among these eleven patients, headache $(n=4,30.8 \%)$ and vomiting $(n=4,30.8 \%)$ were the most common presentations at relapse, followed by paraparesis $(n=2,15 \% .4)$ and ataxia $(n=1,7.8 \%)$. The cerebrospinal fluid (CSF) cytology was not routinely tested. CNS relapsed NB was confirmed by pathology in four patients, and the detailed histological examination excluded the diagnosis of a second primary CNS malignancy.

Among the eleven patients, five did not receive any treatment at relapse and finally died of the disease. Curative treatments were tried in six patients (No.3, No. 4, No. 7, No. 8, No. 9 and No. 11), who received oral administration of temozolomide at $150 \mathrm{mg} / \mathrm{m}^{2}$ followed by intravenous injection of topotecan at $0.75 \mathrm{mg} / \mathrm{m}^{2}$ for five consecutive days at intervals of 21-28 days. More details about treatments for the six patients are presented in Table 2. Among these six assessable patients, one (NO.3) had parenchyma relapse alone, who showed stable disease (SD) after 5 cycles of chemotherapy and withdrew from the treatment. However, he was alive with tumor at the last follow-up. Unbelievably, he survived for more than six years. Moreover, another two patients (No. 4, No. 11) achieved SD either after 4 or 3 cycles of chemotherapy, but they died of the disease soon after treatment withdrawal. Two patients (No. 7, No. 8) attained partial remission, underwent surgery after 4 cycles of chemotherapy, and received 4 cycles of postoperative chemotherapy and radiation. Patient No.7 with parenchyma relapse showed no evidence of disease at 19 months. Patient No.8 had relapsed disease again but was still alive. Patient No. 9 showed progressive disease (PD) after 4 cycles of chemotherapy and died finally.

\section{Toxicity}

A total of 32 courses of chemotherapy were administered to the six patients. On the whole, both topotecan and temozolomide were well tolerated. No toxicity-related death was reported. All patients experienced grade 2 or 4 neutropenia in the course of treatment. No grade 3-4 infection or mucositis was noted. Meanwhile, grade 2 vomiting was a common presentation in the course of treatment. 


\section{Discussion}

It should be noted that, due to the lack of experience, imaging evaluation of disease progression or recurrence in the CNS was not routinely performed at the early stage of this retrospective study until two patients (No.5 and No. 6) suffered from CNS relapse immediately after the completion of delayed intensification chemotherapy at our institution. Historically, CNS involvement in NB is quite rare, which is usually a manifestation of end-stage disease because CNS is a sanctuary site for NB cells, since many chemotherapy and immunotherapy agents can not cross the blood-brain barrier.[1, 11].

The median time from the completion of chemotherapy to CNS relapse was 5 months in the present retrospective study, while that was reported to be 12 months at the Memorial Sloan-Kettering Cancer Center (MSKCC)[12] and 14 months at the Institut Curie and the Institut Gustave-Roussy[11]. Actually, in a larger case series, the median time from initial diagnosis to CNS relapse was fairly consistent (13 months). However, to our surprise, all the eleven patients had relapsed disease during the treatment of oral 13-cis retinoic acid. Moreover, eight of the eleven $(72.7 \%)$ patients suffered from relapse within no more than 6 months after the completion of delayed intensification chemotherapy. All the eleven patients, except for patients No.5 and No. 6, received head MRI after chemotherapy and clear CNS involvements were shown. From then on, head MRI was re-examined at intervals of three months. The precise cause of rapid CNS relapse in these patients remained unclear. The most probable explanation was that autologous hematopoietic progenitor cell transplantation was not included in the standard therapy for high-risk NB patients due to the financial burdens, and no patient received myeloablative treatment. Besides, anti-GD2 antibody is not available in China, except for Hong Kong. Alternatively, it is also suggested that, patients diagnosed after 1990 and treated with the more intensive induction therapies have a higher incidence of CNS metastasis than those diagnosed earlier[12]. Also, it is suggested that the strict monitoring policy facilitates to detect relapse early, when the tumor burden is still small. Further, it is hypothesized that, when extensive or bulky disease is involved in relapse, prolonged survival and even radical cure are less likely. Undoubtedly, MIBG is the most sensitive surveillance for detecting high-risk NB relapse[2, 13]. However, MIBG is not available, which makes it difficult to surveille CNS relapsed NB.

In some study, CNS involvement in NB may be ascribed to the barrier action of the dura that protects CNS parenchyma from cell invasion[14]. In other opinions, CNS NB may result from the direct extension of neuroblasts from bone or bone marrow[15]. In our series, five of the eleven patients developed isolated CNS relapsed NB. As reported in literature, the CNS was the first or only site of PD in several patients[3, 14]. However, in our study, four of the five patients with isolated CNS relapse without overlying bony disease occurred in parenchyma, while the disease in the remaining one patient took place in leptomeninges. Thus, it is hypothesized that, neuraxis involvement may occur by the hematologic route during the active disease period, but the later growth and intra-CNS dissemination via the CSF are not consistent with our observation. Collectively, it can hardly determine whether the CNS relapsed NB occurs by breaking down the blood-brain barrier or by hematogenous spreading.

To the best of our knowledge, no consensus is reached about how to manage CNS relapse in NB patients so far. A study from MSKCC suggests that, it is an important part of management to target the entire neuraxis with craniospinal irradiation (CSI) rather than delivering focal radiotherapy[16]. Also, it has been proved that CSI is effective on CNS relapse, but carries significant long-term morbidity. Nowadays, MSKCC recommends 18 Gy as the new standard CSI dose for CNS relapsed NB in conjunction with compartmental radioimmunotherapy (RIT) and CNS penetrating chemotherapy[1]. Again, due to the limited availability of RIT, this treatment regimen is not prevalent.

Among the six patients who received treatment, two with isolated CNS relapsed NB survived after follow-up for 60 and 19 months, respectively. Interestingly, patient No.3 received 5 cycles of chemotherapy after relapse without further treatment, as required by his parents, and he was alive with tumor for more than 6 years. His young age and non-bone marrow involvement at diagnosis might be the best explanation for this. At the same time, the other alive patient had no metastasis disease at diagnosis; actually, she had stage III disease with unfavorable shimada classification. Besides, both of these two patients had CNS relapse alone. 
It seemed that patients with isolated CNS relapse had superior outcomes. Furthermore, it was found in our retrospective study that, temozolomide and topotecan were well tolerated, with the major treatmentrelated adverse events of manageable hematologic toxicities. Typically, it is hard to assess the effectiveness of temozolomide and topotecan for CNS relapsed NB due to the limited sample size. However, as shown by a phase II study from SIOP regarding the use of temozolomide combined with topotecan in treating relapsed or refractory $\mathrm{NB}$, the tumor control rate (complete response $(\mathrm{CR})+$ partial response $(\mathrm{PR})+$ mixed response $(\mathrm{MR})+\mathrm{SD})$ is $68 \%$ (95\% confidence interval (CI), 63-90\%). In the meantime, the 12-month progression-free survival (PFS) and OS are 42\% and 58\%, respectively[17]. Furthermore, another phase II trial shows that the overall objective response rate (ORR) is $28 \%$ (95\% CI, 12.7-47.2) in medulloblastoma and $10 \%(95 \%$ CI, 0.3-44.5) in primitive neuroectodermal tumors treated with temozolomide and topotecan[18]. Overall, the temozolomide-topotecan combination achieved comparable therapeutic effect to other combinations on treating children with heavily pretreated CNS relapsed NB in our retrospective study. Therefore, this combination may likely be utilized further in front line or other salvage therapies to treat CNS relapsed NB.

In conclusion, the median time from the completion of chemotherapy to CNS relapse is very short, as reported in our retrospective study. It seems that patients with isolated CNS relapsed disease have better outcomes. We suggest the temozolomide-topotecan combination as an add-one backbone for further salvage treatment in children with CNS relapsed NB, although this combination is not superior to others.

Conflict of Interest statement : The authors indicated no potential conflicts of interest.

\section{References}

1. Luo LY, Kramer K, Cheung NV, Kushner BH, Modak S, Basu EM, Roberts SS, Wolden SL: Reduceddose craniospinal irradiation for central nervous system relapsed neuroblastoma . Pediatr Blood Cancer2020:e28364.

2. Owens C, Li BK, Thomas KE, Irwin MS: Surveillance imaging and radiation exposure in the detection of relapsed neuroblastoma .Pediatr Blood Cancer 2016, 63 (10):1786-1793.

3. Blatt J, Fitz C, Mirro J, Jr.: Recognition of central nervous system metastases in children with metastatic primary extracranial neuroblastoma . Pediatric hematology and oncology 1997,14 (3):233-241.

4. Kramer K, Kushner B, Heller G, Cheung NKV: Neuroblastoma metastatic to the central nervous system. The Memorial Sloan-kettering Cancer Center Experience and A Literature Review . Cancer 2015,91 (8):1510-1519.

5. Perisa MP, Storey M, Streby KA, Ranalli MA, Skeens M, Shah N:Cabozantinib for relapsed neuroblastoma: Single institution case series . Pediatr Blood Cancer 2020, 67 (7):e28317.

6. Brisse HJ, McCarville MB, Granata C, Krug KB, Wootton-Gorges SL, Kanegawa K, Giammarile F, Schmidt M, Shulkin BL, Matthay KK et al : Guidelines for imaging and staging of neuroblastic tumors: consensus report from the International Neuroblastoma Risk Group Project . Radiology 2011, 261 (1):243-257.

7. Brodeur GM, Pritchard J, Berthold F, Carlsen NL, Castel V, Castelberry RP, De Bernardi B, Evans AE, Favrot M, Hedborg F et al : Revisions of the international criteria for neuroblastoma diagnosis, staging, and response to treatment . Journal of clinical oncology : official journal of the American Society of Clinical Oncology 1993, 11 (8):1466-1477.

8. Park JR, Kreissman SG, London WB, Naranjo A, Cohn SL, Hogarty MD, Tenney SC, Haas-Kogan D, Shaw PJ, Kraveka JM et al :Effect of Tandem Autologous Stem Cell Transplant vs Single Transplant on Event-Free Survival in Patients With High-Risk Neuroblastoma: A Randomized Clinical Trial . Jama 2019,322 (8):746-755. 
9. Zhang YT, Chang J, Xu HM, Li YN, Zhong XD, Liu ZL: Treatment of Neuroblastoma with a Novel Delayed Intensification Chemotherapy .Indian J Pediatr 2019, 86 (2):126-131.

10. Schmidt M, Simon T, Hero B, Schicha H, Berthold F: The prognostic impact of functional imaging with (123)I-mIBG in patients with stage 4 neuroblastoma $>1$ year of age on a high-risk treatment protocol: results of the German Neuroblastoma Trial NB97 .European journal of cancer (Oxford, England : 1990) 2008,44 (11):1552-1558.

11. Matthay KK, Brisse H, Couanet D, Couturier J, Bénard J, Mosseri V, Edeline V, Lumbroso J, ValteauCouanet D, Michon J: Central nervous system metastases in neuroblastoma: radiologic, clinical, and biologic features in 23 patients . Cancer 2003,98 (1):155-165.

12. Kramer K, Kushner B, Heller G, Cheung NK: Neuroblastoma metastatic to the central nervous system. The Memorial Sloan-kettering Cancer Center Experience and A Literature Review . Cancer 2001,91 (8):1510-1519.

13. Kushner BH, Kramer K, Modak S, Cheung NK: Sensitivity of surveillance studies for detecting asymptomatic and unsuspected relapse of high-risk neuroblastoma. Journal of clinical oncology : official journal of the American Society of Clinical Oncology 2009,27 (7):1041-1046.

14. Kellie SJ, Hayes FA, Bowman L, Kovnar EH, Langston J, Jenkins JJ, 3rd, Pao WJ, Ducos R, Green AA: Primary extracranial neuroblastoma with central nervous system metastases characterization by clinicopathologic findings and neuroimaging . Cancer 1991,68 (9):1999-2006.

15. Banerjee S, Marwaha RK, Bajwa RP: Primary pelvic neuroblastoma with central nervous system metastases . Pediatric hematology and oncology 1995, 12 (3):309-312.

16. Croog VJ, Kramer K, Cheung NK, Kushner BH, Modak S, Souweidane MM, Wolden SL: Whole neuraxis irradiation to address central nervous system relapse in high-risk neuroblastoma . International journal of radiation oncology, biology, physics 2010, 78 (3):849-854.

17. Di Giannatale A, Dias-Gastellier N, Devos A, Mc Hugh K, Boubaker A, Courbon F, Verschuur A, Ducassoul S, Malekzadeh K, Casanova M et al : Phase II study of temozolomide in combination with topotecan (TOTEM) in relapsed or refractory neuroblastoma: a European Innovative Therapies for Children with Cancer-SIOP-European Neuroblastoma study . European journal of cancer (Oxford, England : 1990) 2014, 50 (1):170-177.

18. Le Teuff G, Castaneda-Heredia A, Dufour C, Jaspan T, Calmon R, Devos A, McHugh K, Leblond P, Frappaz D, Aerts I et al : Phase II study of temozolomide and topotecan (TOTEM) in children with relapsed or refractory extracranial and central nervous system tumors including medulloblastoma with post hoc Bayesian analysis: A European ITCC study .Pediatr Blood Cancer 2020, 67 (1):e28032.

Hosted file

Table one new.pdf available at https://authorea.com/users/375574/articles/492752-relapsedneuroblastoma-in-the-central-nervous-system-a-single-center-retrospective-study

Hosted file

Table two.pdf available at https://authorea.com/users/375574/articles/492752-relapsedneuroblastoma-in-the-central-nervous-system-a-single-center-retrospective-study 